\title{
Abnormal Cytolytic Activity of Lymphocyte Function-associated Antigen-1-deficient Human Cytolytic T Lymphocyte Clones
}

\author{
Steven J. Mentzer, Barbara E. Bierer, Donald C. Anderson, Timothy A. Springer, and Steven J. Burakoff \\ Divisions of Pediatric Oncology and Membrane Immunochemistry, Dana-Farber Cancer Institute, Harvard Medical School, \\ Boston, Massachusetts 02115; and Department of Medicine, Baylor University, Houston, Texas 77030
}

\begin{abstract}
The involvement of the lymphocyte function-associated antigen1 (LFA-1) membrane molecule in cytolytic T lymphocyte (CTL) interactions with lymphoid target cells was investigated using CTL clones derived from two patients with a heritable deficiency of LFA-1. LFA-1 surface expression on the CTL clones was $1 \%$ of the normal level of LFA-1, unchanged with prolonged culture, and identical on 14 different CTL clones. The function of the LFA-1 molecule was addressed using the LFA-1-deficient CTL clones and LFA-1-deficient lymphoid target cells. The lytic activity of the LFA-1-deficient CTL clones was $43 \%$ of control when tested against a target cell line expressing normal levels of LFA-1 and $<10 \%$ of control when tested against an LFA-1deficient target cell line. These results demonstrate a direct involvement of LFA-1 in CTL-mediated cytolysis and suggest a more general dependence on LFA-1 in lymphoid cell-cell interactions.
\end{abstract}

\section{Introduction}

Human lymphocyte function-associated antigen-1 (LFA-1) ${ }^{1}$ is a $177-$ and $95-\mathrm{kD}$ glycoprotein heterodimer that is expressed on all leukocytes, most thymocytes, and on a third of bone marrow cells (1). The LFA-1 molecule was initially identified by screening monoclonal antibodies (MAb) for their ability to inhibit cytolytic $\mathrm{T}$ lymphocyte (CTL)-mediated cytotoxicity in the absence of complement $(1,2)$. MAb to LFA-1 significantly inhibited not only cell-mediated cytotoxicity but $\mathrm{T}$ cell proliferation as well (1). In contrast, MAb to a variety of other cell surface molecules, often present at higher cell surface density than LFA-1, did not inhibit $T$ cell function. The ability of anti-LFA-1 MAb to inhibit a variety of $T$ cell functions led to the suggestion that LFA-1 was an essential cell-cell interaction molecule.

A unique opportunity to assess the function of the LFA-1 molecule has been provided by patients with a genetic deficiency of the LFA-1 glycoprotein. The LFA-1 deficiency syndrome is an immunodeficiency characterized by a susceptibility to re-

Address correspondence to Dr. Mentzer, 1630D, Dana-Farber Cancer Institute, 44 Binney St., Boston, MA 02115.

Received for publication 8 May 1986.

1. Abbreviations used in this paper: CR3, complement receptor type 3; CTL, cytolytic T lymphocyte; EBV, Epstein-Barr virus; F-GAM, fluorescein-conjugated $\mathrm{F}\left(\mathrm{ab}^{\prime}\right)_{2}$ goat anti-mouse antibody; IL-2, interleukin 2; LFA-1, lymphocyte function-associated antigen-1; LU, lytic units; MAb, monoclonal antibodies.

J. Clin. Invest.

(C) The American Society for Clinical Investigation, Inc.

$0021-9738 / 86 / 11 / 1387 / 05 \quad \$ 1.00$

Volume 78, November 1986, 1387-1391 current life-threatening infections (3). Biochemical analysis of the leukocytes from LFA-1-deficient patients have shown a deficiency of not only LFA-1, but of a structurally related family of molecules (4). LFA-1, complement receptor type 3 (CR3), and p150,95 are heterodimers with different heavy chains but a structurally identical $95-\mathrm{kD}$ light chain. All three molecules are deficient in patients with the clinical syndrome (5). The advantage of studying CTL from these deficient patients is that significant levels of CR3 and p150,95 are not expressed on normal $T$ cells. Thus, the CTL from these patients have a selective deficiency of the LFA-1 membrane molecule.

Previous in vitro studies of LFA-1-deficient CTL function have been inconclusive. Arnaout et al. (6) and Miedema et al. (7) have found no significant defect in CTL function. Krensky et al. (8), using HLA-typed related and unrelated controls, demonstrated abnormalities in both cell-mediated cytotoxicity and T cell proliferation. Similarly, Fischer et al. (9) have found severely depressed cell-mediated immune function. The inconsistencies observed in vitro may be explained by the use of polyclonal $\mathrm{T}$ cell responder populations to assess CTL function. Bulk cultures, even from the same individual, can demonstrate significant differences in cytolytic activity (10). Therefore, a comparison of clones directed at the same alloantigen should provide a more definitive evaluation of cytolytic activity.

In this report, we used the genetic deficiency of LFA-1 to investigate the role of LFA-1 in CTL-target cell interactions. The deficiency of LFA-1 on CTL clones was associated with only a $43 \%$ decrease in the lysis of a lymphoid target cell due to the ability of these CTL clones to utilize target cell LFA-1. When LFA-1 was deficient on both the CTL and the target cell, lytic activity was decreased by $>90 \%$. These results indicate a direct involvement of LFA-1 in CTL-mediated cytolysis, and suggest possible mechanisms for CTL compensation in the LFA-1 deficiency syndrome.

\section{Methods}

Peripheral blood lymphocytes. Peripheral blood lymphocytes were obtained from LFA-1-deficient patient 6 and his son (patient 8). Control lymphocytes were obtained from a healthy donor (Col). Lymphocytes obtained from $\mathrm{Col}$ demonstrated a normal response in vitro based on a variety of functional and phenotypic assays (10). HLA typing was performed by the Immunology Research Laboratory at Baylor University and the Histocompatibility Laboratory at the Dana-Farber Cancer Institute: patient 6 (HLA-A1, B8, B14, DR3, DR7), patient 8 (HLA-A1, A32, B8, Bw61, DR3, DRw6), and Col (HLA-A11, A32, B27, Bw51, DR7).

Culture medium. Cells were grown in culture medium consisting of RPMI 1640 (M. A. Bioproducts, Walkersville, MD) supplemented with $10 \%$ heat-inactivated fetal calf serum (Gibco, Grand Island, NY), 100 $\mathrm{U} / \mathrm{ml}$ penicillin (Gibco), $100 \mu \mathrm{g} / \mathrm{ml}$ streptomycin (Gibco), $10 \mathrm{mM}$ Hepes (Sigma Chemical Co., St. Louis, MO), and $25 \mu \mathrm{M}$ 2-mercaptoethanol (Eastman Organic Chemicals, Rochester, NY). Long-term CTL cultures and CTL clones were maintained in $10 \%$ human conditioned medium. 
Interleukin 2 (IL-2) containing human conditioned medium. The method for IL-2 production has been described elsewhere (10). Briefly, peripheral blood leukocytes obtained from five different platelet donors were mixed and washed three times. The cells were resuspended at a concentration of $3 \times 10^{6} \mathrm{cells} / \mathrm{ml}$ in RPMI 1640 (M. A. Bioproducts) containing 3\% fetal calf serum and $0.15 \%$ phytohemagglutinin (Bacto PHA-P; Difco Laboratories, Detroit, MI). Special additives included 2.8 $\mu \mathrm{M}$ indomethacin, $3 \mathrm{mM}$ lithium chloride, and $50 \mu \mathrm{M}$ hydroxyurea, all from Sigma Chemical Co. The cells were cultured for $72 \mathrm{~h}$ and the supernatants were collected. The supernatants were ammonium sulfateprecipitated $(50 \%, 75 \%)$ and dialyzed. The purified conditioned medium was filter sterilized and stored at $-4^{\circ} \mathrm{C}$.

Cytotoxicity assay. CTL assays were performed in triplicate in Vbottom microtiter wells (Linbro, Flow Laboratories, Hamden, CT). Effector cells were added in threefold dilutions. Target cells, preincubated in $0.1 \mathrm{mCi}$ of ${ }^{51} \mathrm{Cr}\left(\mathrm{Na}^{51} \mathrm{CrO}_{4}\right.$, New England Nuclear, Boston, $\left.\mathrm{MA}\right)$ for $2 \mathrm{~h}$ and washed three times, were added at $10^{3}$ cells/well. Microtiter plates were centrifuged and incubated at $37^{\circ} \mathrm{C}$ for $4 \mathrm{~h}$. After incubation, the plates were again centrifuged and the supernatants assayed for ${ }^{51} \mathrm{Cr}$ release. Specific cytotoxicity was calculated as percent cytotoxicity $=100$ $\times$ (cpm experimental release - $\mathrm{cpm}$ spontaneous release)/(total cpm - cpm spontaneous release). When blocking monoclonal antibodies were used, they were added to the wells at the start of the assay. The standard deviation of the triplicate wells rarely exceeded $2-4 \%$ of the specific lysis. Lytic units (LU) were calculated as the number of effector cells required for $50 \%$ lysis of $10^{3}$ target cells. $L U$ were based on effector titrations and confirmed with the following formula: $\mathrm{LU} / 10^{3}=10^{3} /$ (effector cells/target cells) $\times[$ (plateau cpm - sample cpm)/(cpm sample) $]$.

CTL clones. Peripheral blood mononuclear cells were separated on a Ficoll/Hypaque gradient (Lymphocyte Separation Medium, Bionetics, Kensington, MD). The mononuclear cells at $2 \times 10^{6}$ cells $/ \mathrm{ml}$ were cocultured with irradiated $\mathrm{JY}$ at $1 \times 10^{5}$ cells $/ \mathrm{ml}$ in 2-ml wells (Linbro) at $37^{\circ} \mathrm{C}$ in a $5 \% \mathrm{CO}_{2}$ incubator. The bulk culture was maintained in $9.6-$ $\mathrm{cm}^{2}$ wells (Nunc, Vangard, Neptune, NJ) by stimulation every 1-2 wk with irradiated stimulator cells. After 6 wk in culture, the cells were again isolated using a Ficoll-Hypaque gradient and cloned using limiting dilution in 96-well round-bottom microtiter plates (Linbro) with $2 \times 10^{4}$ irradiated JY cells as a feeder layer. Culture medium containing $10 \%$ human conditioned medium was used for cloning; fresh medium was added every $3 \mathrm{~d}$. Clones were obtained at a concentration of $1 \mathrm{cell} / 10$ wells. The clones chosen for further analysis were subcloned at $<1$ cell/ well. The specificity of each clone was determined using a panel of HLAtyped target cells and monoclonal antibody blocking. Col clones 350 , 349, and 354 were specific for HLA-A2; clones 147, 303, and 353 were specific for HLA-B7. The CTL line MMJ was specific for the cell line JY. These clones were shown to have comparable lytic activity in comparisons with clones obtained from two other normal donors.

Cell surface immunofluorescence. Cells were washed twice with phosphate-buffered saline containing $2.5 \%$ fetal calf serum and $0.02 \%$ sodium azide. Approximately $10^{6}$ cells were incubated on ice for $30 \mathrm{~min}$ with an excess concentration of monoclonal antibody. The cells were washed twice and stained with fluorescein-conjugated goat $F\left(a b^{\prime}\right)_{2}$-antimouse (F-GAM) IgG antibody (Tago Inc., Burlingame, CA) diluted 1:10. The cells were incubated on ice for another $30 \mathrm{~min}$. After three washes, the cells were fixed in $1 \%$ paraformaldehyde and analyzed on a FACS I (Becton-Dickinson \& Co., Mountain View, CA).

Monoclonal antibodies. MAb to LFA-1 were derived from subcloned hybridomas that produced antibodies to LFA-1 as previously described (2). MAb to the LFA-1 alpha chain were TS1/22, TS2/14, TS1/11, and TS2/4. MAb to the LFA-1 beta chain was TS1/18. Titration curves were used to establish saturating concentrations of MAb. The HLA-A2-specific MAb PA2.1 was described elsewhere (11).

Tumor cell lines. The human tumor cell lines were maintained in culture media and regularly passaged. All lines were typed by the HLA tissue-typing laboratory, Dana-Farber Cancer Institute: JY (HLA-A2, B7, DR4, DR6), Priess (HLA-A2, B15, DR4), 23.1 (HLA-A2, B27, DR8), AS (HLA-A2, A24, B27, DR4), PGF (HLA-A3, B7, DR2), Thal B (HLAA3, A10, B7, B51, DR2, DR5), Daudi (HLA- -, -, DR6), and BBN (HLA-A2, A32, B35, B51, DR3, DR4).

\section{Results}

LFA-1 surface expression. Long-term $\mathrm{T}$ cell lines from patients 6 and 8 of the Texas series (8) as well as parallel control lines (Col) were maintained for $>6$ mo in culture. Immunofluorescence analysis of the LFA-1 alpha and beta subunits demonstrated that the $\mathrm{T}$ lymphocytes of moderately deficient patients' 6 and 8 (3) expressed $\sim 1 \%$ of the normal amount of cell surface LFA-1. Serial immunofluorescence of the $T$ cell lines revealed no change in the level of LFA-1 expression after 6 mo of in vitro culture. The expression of LFA-1 also did not change after treatment with IL-2 or interferon-gamma (not shown). To identify any clonal heterogeneity in the expression of LFA-1, 14 CTL clones were investigated using a similar immunofluorescence analysis. These clones were derived from six distinct bulk cultures from both patient 6 and patient 8 . The 14 clones demonstrated an identical level of LFA-1 expression. Staining of a representative clone is shown in Fig. 1. Surface expression of LFA-1 on the CTL clones was also the same level as on the bulk CTL population. Thus, no heterogeneity of LFA-1 surface expression was observed on the LFA-1-deficient CTL.

Lytic activity of LFA-1-deficient CTL clones. The genetic deficiency of LFA-1 permitted the assessment of CTL interactions with lymphoid target cells when LFA-1 was (a) deficient on the CTL, $(b)$ deficient on the target cell, or $(c)$ deficient on both the CTL and target cell. In normal circumstances, LFA-1 is present on all lymphocytes. Although LFA-1 is expressed on both CTL and lymphoid target cells, the CTL-target cell interaction appears to depend primarily on the LFA-1 expressed on the CTL. MAb pretreatment of the effector cell maximally inhibits cytolysis, whereas MAb pretreatment of the target cell has minimal effect (1). Despite the significant deficiency of LFA-1, detailed analysis of six HLA-A2-specific CTL clones showed that the LFA-1-deficient clones were capable of effectively lysing a variety of lymphoid target cells (Table I). Based on 26 separate experiments, the lytic activity of the LFA-1-deficient CTL clones (as quantified by lytic units) was $43 \%$ of control CTL clones (Table II).

The effective lysis of lymphoid target cells suggested that LFA-1-deficient CTL could compensate for their deficiency. The molecular basis for this compensation was unclear. The LFA1-deficient CTL clones did not express an increased number of antigen nonspecific or "accessory" molecules that have been shown to participate in the CTL-target cell interaction. The
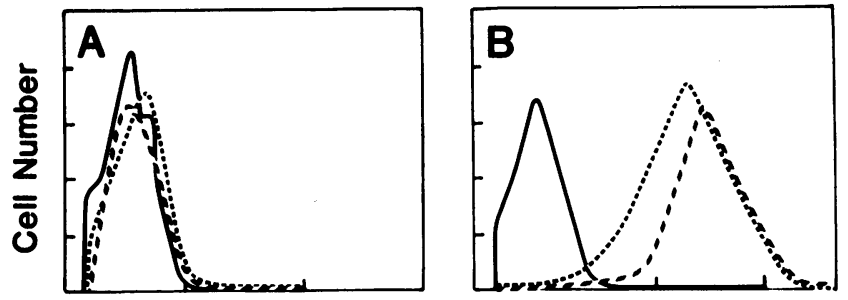

Log Fluorescence Intensity

Figure 1. Surface expression of LFA-1 alpha and beta chains on patient 6 (clone F4) $(A)$ and Col (clone 350) (B) CTL clones. LFA-1 alpha chain was stained with a mixture of anti-alpha chain MAb (Ts1/ 22, Ts1/11) and F-GAM antibody (-----). The LFA-1 beta chain was stained with TS1/18 and F-GAM antibody (-- - - ). Negative controls were stained with F-GAM antibody alone (-). The anti-LFA1 MAb were used at a 1:200 dilution of hybridoma ascites. 
Table I. Specificity of LFA-1-deficient Clones

\begin{tabular}{|c|c|c|c|c|c|c|c|}
\hline \multirow[b]{2}{*}{ Clone‡ } & \multicolumn{7}{|c|}{ \% Specific lysis* } \\
\hline & $\begin{array}{l}\text { Priess } \\
\text { (A2) }\end{array}$ & $\begin{array}{l}23.1 \\
\text { (A2) }\end{array}$ & $\begin{array}{l}\text { AS } \\
\text { (A2) }\end{array}$ & $\begin{array}{l}\text { JY } \\
(A 2, B 7)\end{array}$ & $\begin{array}{l}\text { PGF } \\
\text { (B7) }\end{array}$ & $\begin{array}{l}\text { Thal B } \\
\text { (B7) }\end{array}$ & $\begin{array}{l}\text { Daudi } \\
\text { (DR6) }\end{array}$ \\
\hline F4 & 64 & 46 & 69 & 84 & 13 & 4 & 4 \\
\hline 49 & 48 & 56 & 51 & 68 & 0 & 0 & 0 \\
\hline 50 & 48 & 41 & 54 & 64 & 0 & 0 & 0 \\
\hline 51 & 71 & 60 & 66 & 85 & 0 & 0 & 0 \\
\hline 52 & 47 & 63 & 55 & 80 & 4 & 1 & 0 \\
\hline 53 & 44 & 49 & 58 & 66 & 5 & 3 & 0 \\
\hline
\end{tabular}

* Percent specific ${ }^{51} \mathrm{Cr}$ release of the indicated target cell lines in a 4-h assay. Complete HLA types are listed in Methods. Relevant HLA types are noted in parentheses.

$¥$ Clone $\mathrm{F} 4$ is derived from patient 6 ; clones $49-53$ are from patient 8 . Effector/target ratios ranged from 6:1 to 9:1.

surface expression of antigen nonspecific molecules such as CD3, CD2, and CD8 was comparable on LFA-1-deficient and control CTL clones (data not shown). Another possibility suggested by MAb pretreatment experiments (8) was that LFA-1-deficient CTL clones were able to utilize LFA-1 expressed on the target cell. To investigate this possibility, B cells from patient 1 of the Texas series (8) were transformed by Epstein-Barr virus (EBV) to obtain a suitable target cell line. The B lymphoblastoid cell line derived from patient 1 (BBN) expressed $\sim 2 \%$ of the LFA1 and $91 \%$ of the HLA-A2 expressed on the EBV-transformed cell line JY (Table III). CTL clones specific for HLA-A2 were tested for lytic activity against JY and the LFA-1-deficient target cell BBN. Calculation of LU indicated that the lytic activity on the LFA-1-deficient target BBN by the LFA-1-deficient CTL clones was $<10 \%$ of CTL clones expressing normal levels of LFA-1 (Table IV). This significant decrease in lytic activity was found with all LFA-1-deficient clones tested.

Utilization of the LFA-1 molecule. The diminished ability to lyse LFA-1-deficient target cells demonstrated that LFA-1-

Table II. Lytic Activity against the Target Cell JY*

\begin{tabular}{|c|c|c|c|}
\hline \multicolumn{2}{|c|}{ Control } & \multicolumn{2}{|c|}{ LFA-1-deficient } \\
\hline Clone & LU¥ & Clone & LU¥ \\
\hline 350 & 363 & F4 & 147 \\
\hline 349 & 370 & 49 & 158 \\
\hline 354 & 394 & 50 & 160 \\
\hline 303 & 416 & 51 & 186 \\
\hline 353 & 305 & 52 & 139 \\
\hline \multirow[t]{2}{*}{ MMJ } & $\underline{270}$ & 53 & $\underline{122}$ \\
\hline & $\bar{X}=353 \S$ & & $X=152$ \\
\hline
\end{tabular}

\footnotetext{
* The target cell in all cases was the JY cell line. Control CTL were established from Col. MMJ was a long-term CTL line. LFA-1-deficient CTL clones were obtained from patient $6(\mathrm{~F} 4)$ and patient 8 (49-53).

$\ddagger L U$ represents the mean $L U$ of at least three separate experiments. LU were calculated as described in Methods.

$\S$ Lytic activity of control and LFA-1-deficient CTL clones were significantly different, $P<0.01$, as assessed by the Wilcoxon rank sum test.
}

Table III. Target Cell Expression of LFA-1 and HLA-A2

\begin{tabular}{lccc}
\hline & \multicolumn{2}{l}{ Mean relative fluorescence intensity* } \\
\cline { 2 - 4 } Target cell & $\begin{array}{l}\text { Anti-alpha } \\
\text { (TS1/22, TS1/11) }\end{array}$ & $\begin{array}{l}\text { Anti-beta } \\
\text { (TS1/18) }\end{array}$ & $\begin{array}{l}\text { Anti-HLA-A2 } \\
\text { (PA2.1) }\end{array}$ \\
\hline BBN & 2.13 & 2.09 & 158.14 \\
JY & 101.58 & 86.97 & 173.04 \\
\hline
\end{tabular}

* The EBV-transformed target cell lines BBN and JY were stained with MAb. A pool of anti-alpha chain MAb, an anti-beta chain MAb, and an anti-HLA-A2 MAb were used at 1:200 dilution of hybridoma ascites and indirectly stained with F-GAM. Control values (F-GAM alone) were subtracted from the mean fluorescence intensity of all three samples.

deficient CTL clones, in contrast to normal CTL, utilized LFA1 expressed on the target cell. The novel utilization of LFA-1 might also involve different functional epitopes on the LFA-1 molecule. To explore functional differences of target cell and CTL LFA-1, we used a panel of MAb to five different epitopes on the LFA-1 molecule. MAb cross-blocking experiments had previously defined different functional epitopes on the LFA-1 molecule (12). The MAb panel, composed of four anti-alpha chain MAb and one anti-beta chain MAb, was used to inhibit killing of the LFA-1 ${ }^{+}$target cell. Both LFA-1-deficient (F4) and control (147) CTL clones demonstrated an identical pattern of MAb inhibition (Table V).

\section{Discussion}

The genetic deficiency of the LFA-1 membrane glycoprotein has provided a unique opportunity to assess the direct involvement of this molecule in CTL function. Six HLA-A2-specific CTL clones from two patients with a deficiency of LFA-1 demonstrated abnormal cytolytic function. The cytolytic activity of these CTL clones was $<50 \%$ of the lytic activity of control CTL clones. When LFA-1 expression was deficient on both the CTL and the target cell, lytic activity was $<10 \%$ of control. These

Table IV. Lytic Activity against the LFA-1-deficient BBN Target Cell*

\begin{tabular}{|c|c|c|c|}
\hline \multicolumn{2}{|l|}{ Control } & \multicolumn{2}{|c|}{ LFA-1 deficient } \\
\hline Clone & LU¥ & Clone & LU¥ \\
\hline 350 & 337 & 49 & 17 \\
\hline 349 & 311 & 50 & 21 \\
\hline 354 & 303 & 51 & 33 \\
\hline \multirow[t]{2}{*}{ MMJ } & $\underline{251}$ & 52 & $\underline{25}$ \\
\hline & $X=301 \S$ & & $\bar{X}=24$ \\
\hline
\end{tabular}

\footnotetext{
* The target cell in all cases was the BBN cell line. BBN was an EBVtransformed cell line from patient 1 . Control CTL clones were established from Col. MMJ was a long-term CTL line. LFA-1-deficient CTL clones 49-52 were established from patient 8.

$¥ \mathrm{LU}$ represents the mean $\mathrm{LU}$ of at least three separate experiments. The variance of lytic activity between experiments was always $<10 \%$. $\S$ Lytic activity of control and LFA-1-deficient CTL were significantly different, $P<0.01$, as assessed by the Wilcoxon rank sum test.
} 
Table V. Inhibition of CTL-mediated

Lysis by a Panel of Anti-LFA-1 MAb*

\begin{tabular}{llll}
\hline & & \multicolumn{2}{l}{ \% Specific lysis } \\
\cline { 3 - 4 } MAbł & Subunit of LFA-1 & Clone F4 & Clone 147 \\
\hline Medium & - & 47 & 64 \\
TS1/18 & Beta & $23(51) \S$ & $26(59)$ \\
TS1/22 & Alpha & $26(46)$ & $25(61)$ \\
TS2 $/ 14$ & Alpha & $27(43)$ & $32(50)$ \\
TS1/11 & Alpha & $40(9)$ & $55(14)$ \\
TS2/4 & Alpha & $47(0)$ & $59(8)$ \\
& & & \\
\hline
\end{tabular}

* Control CTL clone 147 and LFA-1-deficient CTL clone F4 were tested against the B lymphoblastoid target cell JY at an E:T ratio of 18:1 in a standard 4-h ${ }^{51} \mathrm{Cr}$-release assay.

$\ddagger$ MAb were used at a 1:150 dilution of hybridoma ascites.

$\S$ Numbers in parentheses indicate percent inhibition.

observations confirm the presence of abnormal CTL function in the LFA-1 deficiency syndrome. In addition, the LFA-1-deficient CTL clones and target cells studied here provide the clearest available evidence that the LFA-1 membrane molecule directly participates in CTL function, and suggest that LFA-1 may play a more general role in lymphoid cell-cell interactions.

The LFA-1 membrane molecule was initially identified as a function-associated molecule by the ability of anti-LFA-1 MAb to inhibit CTL-mediated lysis. Because LFA-1 is expressed on $\mathrm{T}$ cells as well as lymphoid target cells, MAb pretreatment was required to identify the relative contributions of effector and target cell LFA-1 to the killing interaction. MAb pretreatment demonstrated that anti-LFA-1 MAb inhibited cytolysis at the level of the effector cell (1). The dominant role of effector cell LFA-1 was confirmed using nonlymphoid target cells. Endothelial cells and fibroblasts do not express LFA-1. When used as target cells, endothelial cells and fibroblasts were effectively lysed by human CTL clones, and this lysis was inhibited by anti-LFA-1 MAb.

Previous experiments with LFA-1-deficient CTL and MAb blocking suggested a difference between normal and LFA-1deficient CTL in the utilization of LFA-1 (8). To clarify this finding, we studied target cells genetically deficient in LFA-1. As expected, control CTL clones demonstrated near normal levels of lytic activity on LFA-1-deficient target cells. In contrast, LFA-1-deficient CTL clones were not able to effectively lyse the LFA-1-deficient target cell. These findings demonstrate that target cell LFA- 1 can be utilized by the effector cell. The reason that target cell LFA-1 is not used in the normal CTL-target cell interaction is unclear. The relatively high density of LFA-1 on normal CTL may diminish the contribution of target cell LFA1. The utilization of target cell LFA-1 may simply reflect the relative cell surface density of LFA-1. Alternatively, the ability of CTL to utilize target cell LFA-1 may require the enhanced expression of an as yet unidentified LFA-1 ligand. Regardless of the specific mechanism, these experiments have identified LFA-1 as an essential molecule in lymphoid cell-cell interactions.

The distinction between CTL and target cell LFA-1 suggests that the function of the LFA-1 molecule may depend on whether it is involved at the level of the CTL or the target cell. To explore the functional utilization of the LFA-1 molecule, we used a panel of anti-LFA-1 MAb $(12,13)$. An identical hierarchy of MAb inhibition of cytolysis was observed for both patient and control
CTL clones. These MAb blocking experiments suggest that the LFA-1 molecule retains the same functional epitopes whether it is utilized at the level of the effector or target cell. The comparable utilization of LFA-1 epitopes suggests that LFA-1 mediates a common function for both LFA-1-deficient and control CTL clones.

The ability of anti-LFA-1 MAb to inhibit CTL-target cell conjugates (14) suggested that LFA-1 may regulate cell-cell adhesion. Studies of B cell-B cell $(15,16), T$ cell-endothelial cell (17), T cell-T cell, myeloid cell-myeloid cell $(18,19)$, and $\mathrm{T}$ cell-fibroblast (20) adhesions have supported this interpretation. The decreased ability of LFA-1-deficient CTL to lyse an appropriate target cell may reflect a diminished adhesion between CTL and target cell. The development of sensitive biophysical measures of CTL adhesive strength will be an important contribution to the assessment of LFA-1 function.

The clinical problems in the LFA-1 deficiency syndrome primarily reflect the patients' susceptibility to bacterial infections. The syndrome is characterized by poor wound healing, severe periodontal disease, and recurrent soft tissue infections $(6,7,9)$. These observations, as well as normal serum immunoglobulin levels and relatively normal delayed hypersensitivity reactions, have led to the speculation that granulocyte function is more impaired than T cell function. Only two reported LFA-1-deficient patients have developed significant infectious complications attributable to a cell-mediated immune defect. One patient died of overwhelming picornavirus infection of the tracheobronchial tree (3). The other patient developed cutaneous candidiasis (9).

The failure of most LFA-1-deficient patients to manifest a clinically significant $T$ cell defect suggests that $T$ cells compensate for their deficiency. There are a number of potential mechanisms of CTL compensation. The most important mechanism is that $T$ cells, in contrast to granulocytes, respond to antigenic challenge by clonal expansion. Studies of polyclonal populations of LFA1-deficient CTL suggest that antigenic challenge expands those $\mathrm{T}$ cells with the greatest capacity to proliferate and exhibit cytolytic activity. The molecular basis of this selection is unclear, but may involve an increase in the number and/or the affinity of other, as yet unidentified, cell surface molecules. An unexpected compensatory mechanism demonstrated in this study appears to be the ability of LFA-1-deficient CTL to utilize LFA1 expressed on the target cell. Together, clonal selection and the novel utilization of cell surface molecules appear to be complementary mechanisms by which human $\mathrm{T}$ cells may compensate for significant genetic deficiencies. Further work will be necessary to elucidate the functional relationship of LFA-1 to other T cell surface molecules and to further define the role of LFA-1 in the CTL-target cell interaction.

\section{Acknowledgments}

The authors would like to thank M. A. V. Crimmins and M. J. Dunn for excellent technical assistance and Carolyn Gregory for the preparation of the manuscript.

This work was supported by National Institutes of Health grants CA09280 and CA-34129.

\section{References}

1. Krensky, A. M., F. Sanchez-Madrid, E. Robbins, J. A. Nagy, T. A. Springer, and S. J. Burakoff. 1983. The functional significance, distribution, and structure of LFA-1, LFA-2, and LFA-3: cell surface 
antigens associated with CTL-target interactions. J. Immunol. 131:611616.

2. Sanchez-Madrid, F., A. M. Krensky, C. F. Ware, E. Robbins, J. L. Strominger, S. J. Burakoff, and T. A. Springer. 1982. Three distinct antigens associated with human T lymphocyte-mediated cytolysis: LFA1, LFA-2, and LFA-3. Proc. Natl. Acad. Sci. USA. 79:7489-7493.

3. Anderson, D. C., F. C. Schmalstieg, M. J. Finegold, B. J. Hughes, R. Rothlein, L. J. Miller, S. Kohl, M. F. Tosi, R. L. Jacobs, A. Goldman, W. T. Shearer, and T. A. Springer. 1985. The severe and moderate phenotypes of heritable Mac-1, LFA-1 deficiency: their quantitative definition and relation to leukocyte dysfunction and clinical features. J. Infect. Dis. 152:668-689.

4. Sanchez-Madrid, F., J. A. Nagy, E. Robbins, P. Simon, and T. A. Springer. 1983. A human leukocyte differentiation antigen family with distinct alpha subunits and common beta subunit: the lymphocyte function-associated antigen (LFA-1), the C3bi complement receptor (OKM1/ Macl) and the p150,95 molecule. J. Exp. Med. 158:1785-1803.

5. Springer, T. A., W. S. Thompson, L. J. Miller, F. C. Schmalstieg, and D. C. Anderson. 1984. Inherited deficiency of the Mac-1, LFA-1, p150,95 glycoprotein family and its molecular basis. J. Exp. Med. 160: 1901-1918.

6. Arnaout, M. A., H. Spitz, C. Terhorst, J. Pitt, and R. F. Todd. 1984. Deficiency of a leukocyte surface glycoprotein in two patients with Mol deficiency. J. Clin. Invest. 74:1291-1300.

7. Miedema, F., P. A. F. Tetteroo, F. G. Terpstra, G. Keizer, M. Roos, R. S. Weening, C. M. R. Weemaes, D. Roos, and C. J. M. Melief. 1985. Immunologic studies with LFA-1 and Mol-deficient lymphocytes from a patient with recurrent bacterial infections. J. Immunol. 134:30753081.

8. Krensky, A. M., S. J. Mentzer, C. Clayberger, F. C. Schmalstieg, D. C. Anderson, S. J. Burakoff, and T. A. Springer. 1985. Heritable lymphocyte function-associated antigen-1 deficiency: abnormalities of cytotoxicity and proliferation associated with abnormal expression of LFA-1. J. Immunol. 135:3102-3108.

9. Lisowska-Grospierre, B., M.-C. Bohler, A. Fischer, C. Mawas, T. A. Springer, and C. Giscelli. 1986. Defective membrane expression of the LFA- 1 complex may be secondary to the absence of the $\beta$ chain in a child with recurrent bacterial infection. Eur. J. Immunol. 16:205208.
10. Krensky, A. M., S. J. Mentzer, J. L. Greenstein, M. Crimmins, C. Clayberger, T. A. Springer, and S. J. Burakoff. 1985. Human cytolytic $T$ lymphocyte clones and their function-associated cell surface molecules. In Hybridoma Technology in the Biosciences and Medicine. T. A. Springer, editor. Plenum Press, New York. 559-573.

11. Parham, P., and W. F. Bodmer. 1978. Monoclonal antibody to a human histocompatibility antigen, HLA-A2. Nature (Lond.). 276:397399.

12. Ware, C. F., F. Sanchez-Madrid, A. M. Krensky, S. J. Burakoff, J. L. Strominger, and T. A. Springer. 1983. Human lymphocyte functionassociated antigen-1 (LFA-1): identification of multiple antigenic epitopes and their relationship to CTL-mediated cytotoxicity. J. Immunol. 131: 1182-1187.

13. Mentzer, S. J., A. M. Krensky, and S. J. Burakoff. 1986. Mapping functional epitopes of the LFA-1 glycoprotein: monoclonal antibody inhibition of NK and CTL effectors. Hum. Immunol. In press.

14. Krensky, A. M., E. Robbins, T. A. Springer, and S. J. Burakoff. 1984. LFA-1, LFA-2, and LFA-3 antigens are involved in CTL-target conjugation. J. Immunol. 132:2180-2182.

15. Mentzer, S. J., S. H. Gromkowski, A. M. Krensky, S. J. Burakoff, and E. Martz. 1985. LFA-1 membrane molecule in the regulation of homotypic adhesions of human B lymphocytes. J. Immunol. 135:9-11.

16. Springer, T. A., R. Rothlein, D. C. Anderson, S. J. Burakoff, and A. M. Krensky. 1985. The function of LFA-1 in cell-mediated killing and adhesion: studies on heritable LFA-1, Mac-1 deficiency and p150,95 on lymphoid cell self-aggregation. Adv. Exp. Med. Biol. 184:311-320.

17. Mentzer, S. J., S. J. Burakoff, and D. V. Faller. 1986. T cell adhesion to endothelial cells is regulated by the LFA-1 membrane molecule. J. Cell. Physiol. 126:285-290.

18. Rothlein, R., and T. A. Springer. 1986. The requirement for LFA-1 in homotypic leukocyte adhesion stimulated by phorbol ester. $J$. Exp. Med. 163:1132-1149.

19. Mentzer, S. J., D. V. Faller, and S. J. Burakoff. 1986. Interferongamma induction of LFA-1 mediated homotypic adhesions of human monocytes. J. Immunol. 137:108-113.

20. Dustin, M. L., R. Rothlein, A. K. Bhan, C. A. Dinarello, and T. A. Springer. 1986. Induction by IL 1 and interferon-gamma: tissue distribution, biochemistry, and function of a natural adherence molecule (ICAM-1). J. Immunol. In press. 